\title{
Swift heavy ion irradiation-induced modifications in structural, magnetic and electrical transport properties of epitaxial magnetite thin films
}

\author{
Ravi Kumara) \\ Inter University Accelerator Centre, Aruna Asaf Ali Marg, New Delhi-110067, India \\ M. Wasi Khan and J. P. Srivastava \\ Department of Physics, Aligarh Muslim University, Aligarh-202002, India \\ S. K. Arora, R. G. S. Sofin, R. J. Choudhary, and I. V. Shvets \\ SFI Nanoscience Laboratories, School of Physics, Trinity College, Dublin 2, Ireland
}

(Received 10 January 2006; accepted 8 June 2006; published online 3 August 2006)

The effect of swift heavy ion (SHI) irradiation $(190 \mathrm{MeV} \mathrm{Ag})$ on structural, electrical transport and magnetic properties of epitaxial magnetite $\left(\mathrm{Fe}_{3} \mathrm{O}_{4}\right)$ thin films (thickness $\sim 70 \mathrm{~nm}$ ) grown on $\mathrm{MgO}\langle 100\rangle$ oriented substrate have been investigated. The $\mathrm{x}$-ray diffraction shows that at low fluence values up to $5 \times 10^{11}$ ions $/ \mathrm{cm}^{2}$, the strain in the films is relaxed, whereas, at higher fluence range $1 \times 10^{12}-1 \times 10^{13}$ ions $/ \mathrm{cm}^{2}$, the epitaxial relationship with the substrate is lost along with a phase transformation from magnetite to more oxidized magnetite phase (i.e., maghemite). The Verwey transition temperature measured by electrical transport is found to increase from 109 to $117 \mathrm{~K}$ with the low fluence SHI irradiation, which is related to the irradiation induced strain relaxation and structural modifications. At higher fluences the system did not show Verwey transition and the resistance is also increased. The similar results were obtained by magnetization studies. The observed magnetization at $1 \mathrm{~T}$ field is increased at low fluence suggesting the reduction of areas with frustrated exchange interactions associated with the cationic arrangement at the anti phase boundaries. At higher fluences it decreases monotonically, indicating the emergence of other phases. The observed modifications are explained on the basis of structural strain and disorder induced by swift heavy ions, which lead to modification of the interionic Coulomb potential at octahedral sublattices and bandwidth in this system. (C) 2006 American Institute of Physics.

[DOI: $10.1063 / 1.2222066]$

\section{INTRODUCTION}

Magnetite $\left(\mathrm{Fe}_{3} \mathrm{O}_{4}\right)$, represented as $\mathrm{Fe}^{3+}\left[\mathrm{Fe}^{2+} \mathrm{Fe}^{3+}\right] \mathrm{O}_{4}$, is a half metallic oxide and exhibit well known spinel structure with $F d 3 m$ space group. One third of the Fe ions with valence state +3 occupy the A site, which is tetrahedrally coordinated by four oxygen ions. The remaining two-thirds with equiproportion of +2 and +3 valence state are octahedrally surrounded by six oxygen ions, known as $\mathrm{B}$ site. There is a growing interest in $\mathrm{Fe}_{3} \mathrm{O}_{4}$ thin films due to their favorable Curie temperature $\left(T_{c} \sim 858 \mathrm{~K}\right)$ combined with its high spin polarization ${ }^{1}$ and presence of first order metal-insulator transition at $122 \mathrm{~K}$ (known as Verwey transition). ${ }^{2}$ The Verwey transition temperature $\left(T_{v}\right)$ in the stochiometric bulk $\mathrm{Fe}_{3} \mathrm{O}_{4}$ is a consequence of the band splitting caused by the electronic correlations and electron-phonon interactions. The phase transition in magnetite originates from an ordering of the $\mathrm{Fe}^{2+}$ and $\mathrm{Fe}^{3+}$ ions at the octahedral $\mathrm{Fe}$ sublattice. ${ }^{3,4}$ However, the mechanisms responsible for the transition have been the subject of a great interest from theoretical as well as experimental standpoints. ${ }^{1,5}$ For the basic understanding of Verwey transition, it has been formulated that the interionic Coulomb interactions at octahedral Fe sublattice are playing an important role. Brabers, Walz, and Kronmuller, ${ }^{6,7}$ have

\footnotetext{
${ }^{a)}$ Author to whom correspondence should be addressed; electronic mail: ranade@nsc.ernet.in
}

presented a mean-field approach to Verwey transition based on an effective interionic Coulomb potential and explained the various aspects. In their model they have pointed out that the Verwey transition is strongly dependent upon the bandwidth and interionic Coulomb interaction. The link between the bandwidth and the Verwey transition temperature was incorporated in models explaining enhanced $T_{v}$ values in magnetite (100) surface., ${ }^{5,8}$ Both these parameters (interionic Coulomb interaction and bandwidth) are strongly dependent on structural strain and cation substitution which are determined by the film synthesization processes. The epitaxial $\mathrm{Fe}_{3} \mathrm{O}_{4}$ films grown on $\mathrm{MgO}$ substrate are known to contain anti phase boundaries (APBs). APBs arise from the fact that the lattice constant of magnetite is twice greater than that of the $\mathrm{MgO}$ substrate and from the crystal symmetry of the film being lower than that of the substrate. APBs were observed experimentally by many groups using scanning tunneling microscopy and transmission electron microscopy, ${ }^{9-11}$ which affect the electrical as well as magnetic properties of the films. ${ }^{12-16}$ The Verwey transition temperature and the saturation magnetization strongly depend upon the density of APBs. ${ }^{16}$ To understand the variation of Verwey transition and various processes involved in it, one has to control the APB density, structural strain and stochiometry of magnetite thin films.

Swift heavy ion (SHI) irradiation is known to generate controlled defects, structural disorder and can modify strain. 
It depends upon the electronic energy loss and fluence values in the target materials. ${ }^{17-19}$ In this article, we present our results on effects of SHI irradiation on Verwey transition, magnetization and phase modification in epitaxial $\mathrm{Fe}_{3} \mathrm{O}_{4}$ thin films.

\section{EXPERIMENTS}

The epitaxial thin films of $\mathrm{Fe}_{3} \mathrm{O}_{4}$ (thickness $\sim 70 \mathrm{~nm}$ ) were grown on $\langle 100\rangle$ oriented $\mathrm{MgO}$ single crystal substrate using an oxygen plasma assisted molecular beam epitaxy (MBE) system (DCA MBEM600) with a base pressure of $5 \times 10^{-10}$ Torr. The details of the growth process are given elsewhere. $^{20}$ Reflection high-energy electron diffraction (STAIB Instruments) was used to determine the growth mode and growth rate $(0.3 \AA / \mathrm{s})$ of the films. In order to avoid the effect of sample quality variation, we cut two well characterized $70 \mathrm{~nm}$ thin films of size $10 \mathrm{~mm} \times 10 \mathrm{~mm}$ into four pieces each of $5 \mathrm{~mm} \times 5 \mathrm{~mm}$ size. The samples were irradiated at room temperature with $190 \mathrm{MeV} \mathrm{Ag}$ ions using the 15UD tandem accelerator at the Inter University Accelerator Center, New Delhi, with a range of fluence values (5 $\times 10^{10}-1 \times 10^{13}$ ions $\left./ \mathrm{cm}^{2}\right)$. The irradiation was performed under high vacuum condition (base pressure $<2$ $\times 10^{-6}$ Torr). The incidence angle of the ion beam was kept slightly away from the surface normal of the sample to avoid channeling effects. The ion beam was focused to a spot of $1 \mathrm{~mm}$ diameter and scanned over a $10 \times 10 \mathrm{~mm}^{2}$ area using a magnetic scanner to irradiate the sample uniformly. To understand the SHI irradiation induced structural modifications and phase transitions, the high-resolution $\mathrm{x}$-ray diffraction (HRXRD) measurements were performed using a multicrystal high-resolution x-ray diffractometer, HRXRD (BedeD1, Bede, UK). Monochromatic $\mathrm{Cu} K_{\alpha}(1.54056 \AA)$ radiation with 20 in. beam divergence was obtained in the diffractometer using four $\mathrm{Si}$ channel-cut crystals. The HRXRD in the double axis configuration was performed to confirm the epitaxial relationship of the $\mathrm{Fe}_{3} \mathrm{O}_{4} / \mathrm{MgO}$ heteroepitaxy. The in-plane $\left(a_{\|}\right)$and out-of-plane $\left(a_{\perp}\right)$ lattice parameters were determined from the analysis of $\omega-2 \theta$ rocking curves measured around the symmetric (200) and asymmetric (311) diffraction planes common to the substrate and thin film. Magnetization measurements were performed using an alternating gradient field magnetometer (Micromag3900, Princeton Measurements) with a sensitivity of $10^{-8}$ emu. The magnetization versus field $(M-H)$ loops were measured at room temperature by applying the magnetic field (maximum field of $1 \mathrm{~T}$ ) in the film plane along the $\langle 100\rangle$ direction. The diamagnetic contribution from the $\mathrm{MgO}$ substrate was subtracted from the measured data by performing $M-H$ loop of the $\mathrm{MgO}$ substrate of similar dimensions as that of thin film sample, in the same field range. The uncertainty in measuring the absolute value of magnetization for the films was about $1 \%$. The electrical resistivity measurements were performed using standard four-probe technique in the temperature range $77-300 \mathrm{~K}$. The magnetic domain structures were measured by magnetic force microscopy (MFM) using Digital Nano-Scope-III.

\section{RESULTS AND DISCUSSION}

It is well known that the swift heavy ion (SHI) irradiation can generate controlled defects (point/clusters and columnar), modify the local strain and induce phase transformation in the materials. When a high-energy heavy ion passes through a material, it loses its energy mainly by two ways: elastic process (nuclear energy loss) and inelastic process (electronic energy loss). The nuclear energy loss is dominant in the low-energy range (few hundreds of $\mathrm{keV}$ ) and the electronic energy loss is dominant in high-energy range (10 MeV and above). In the present case, we have used $190 \mathrm{MeV} \mathrm{Ag}{ }^{15+}$ ions on $\mathrm{Fe}_{3} \mathrm{O}_{4}$ thin films. The electronic energy loss, $S_{e}$, nuclear energy loss, $S_{n}$ and the range of ion, $R_{p}$, calculated by using standard SRIM simulation program, ${ }^{21}$ are $25.79 \mathrm{keV} / \mathrm{nm}, 69.24 \mathrm{eV} / \mathrm{nm}$ and $12.3 \mu \mathrm{m}$, respectively. From the calculated values, it is clearly evident that the $S_{n}$ is about three orders of magnitude less than that of $S_{e}$ and the range is much larger than the film's thickness $(70 \mathrm{~nm})$. To create columnar defects in materials a certain threshold value of $S_{e}$ is required ${ }^{22}$ and for bulk $\mathrm{Fe}_{3} \mathrm{O}_{4}$, its value is about $36 \mathrm{keV} / \mathrm{nm}$. In the present case, the $S_{e}$ value is less than the threshold value required to produce columnar defects and we expect that only point defects or clusters of point defects will be produced in $\mathrm{Fe}_{3} \mathrm{O}_{4}$ thin films. It is important to understand here that the way in which the huge energy $\left(S_{e}\right.$ $=25.79 \mathrm{keV} / \mathrm{nm}$ ) is deposited to the system will modify the materials. In general, there are two mechanisms by which the energy can be deposited, thermal spike ${ }^{23}$ and Coulomb explosion. ${ }^{24}$ In the case of the first mechanism, the ion beam will excite the electronic system at the local site and the electrons will transfer their energy to phonon via electronphonon coupling, resulting in an increase of the local temperature. In this way local defects are created or, if the system already contains such defects, then they can be annealed. In the second mechanism, during passage through the material, ions can create an ionization zone along their path. The ionized zone with positive charges may explode under electrostatic force and create the distortion in the materials or alternatively it may induce some type of the strain in the materials. Here again we can say that such additional strain caused by the ionized zone is superimposed on the strain present in the film before the irradiation and therefore the overall strain in the material can be reduced or increased. In this way, we can conjecture that the SHI irradiation can generate strain, release strain, anneal or create the defects depending upon the initial state of the material. We have recently studied the strain status in epitaxial $\mathrm{Fe}_{3} \mathrm{O}_{4}$ films grown on (100) $\mathrm{MgO}$ substrate. ${ }^{25}$ We have established that the films retain the fully strained status at the thickness much in excess of the critical thickness (for $\mathrm{Fe}_{3} \mathrm{O}_{4} / \mathrm{MgO}$ hetroepitaxial system, the estimated critical thickness for strain relaxation on the basis of the widely used Mathew and Blakeslee model is $60 \mathrm{~nm})^{26}$ values. Therefore, the present study exploited the avenue of changing the local strain in the $\mathrm{Fe}_{3} \mathrm{O}_{4}$ film by SHI irradiation.

To understand the structural modifications induced by SHI irradiations, we have performed high-resolution x-ray diffraction measurements. Figures 1(a) and 1(b) show the 

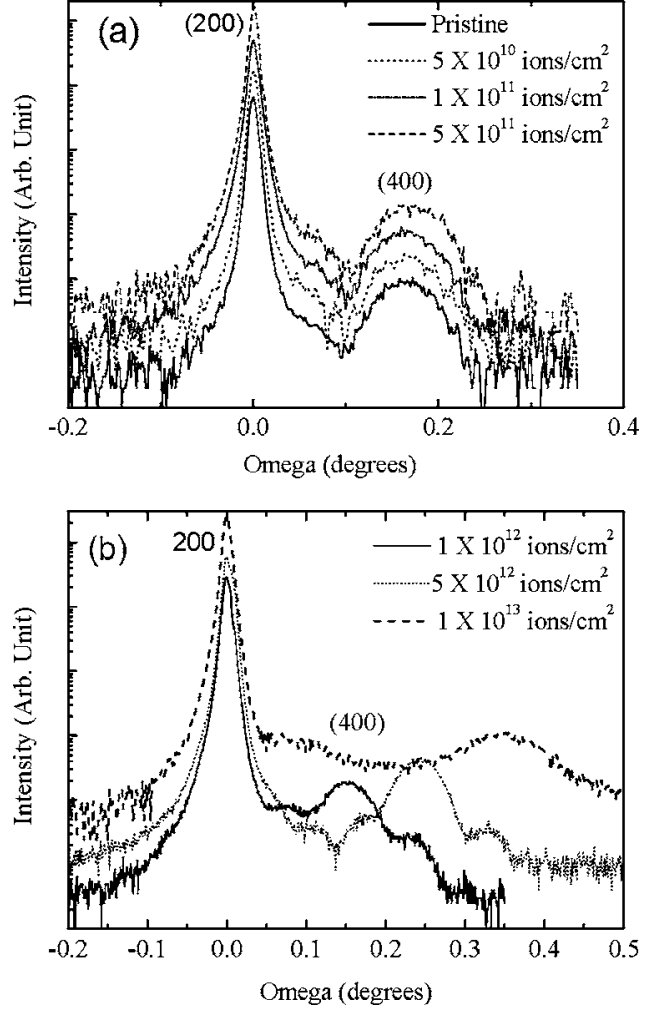

FIG. 1. The $\omega-2 \theta$ rocking curves for pristine and $190 \mathrm{MeV} \mathrm{Ag}^{15+}$ ion irradiated 70-nm-thick $\mathrm{Fe}_{3} \mathrm{O}_{4}$ films (a) with low fluences $\left(5 \times 10^{10}, 1 \times 10^{11}\right.$ and $5 \times 10^{11}$ ions $\left./ \mathrm{cm}^{2}\right)$ and (b) with higher fluences $\left(1 \times 10^{12}, 5 \times 10^{12}\right.$ and 1 $\times 10^{13}$ ions $/ \mathrm{cm}^{2}$ ) measured for (400) Bragg planes of $\mathrm{Fe}_{3} \mathrm{O}_{4}$ common to (200) planes of $\mathrm{MgO}$. The curves are shifted along the vertical axis for clarity.

HRXRD results for (400) Brag plane of $\mathrm{Fe}_{3} \mathrm{O}_{4}$ common to (200) of $\mathrm{MgO}$ for pristine and $190 \mathrm{MeV} \mathrm{Ag}^{+15}$ ion irradiated films with fluence values in the range of $5 \times 10^{10}-1$ $\times 10^{13}$ ions $/ \mathrm{cm}^{2}$. It is clearly evident from Fig. 1(a) that there is no appreciable change in separation between the substrate (200) peak and the (400) peak of $\mathrm{Fe}_{3} \mathrm{O}_{4}$ thin films up to the fluence value $5 \times 10^{11}$ ions $/ \mathrm{cm}^{2}$. However, as the ion fluence is further increased to $1 \times 10^{12}$ ions $/ \mathrm{cm}^{2}$, the HRXRD spectra are modified to a great extent and the (400) peak splits into three peaks which indicates the structural disorder and onset of the phase transition [see Fig. 1(b)]. At higher fluence values such as $1 \times 10^{13}$ ions $/ \mathrm{cm}^{2}$ the (400) peak disappeared and the system transformed from magnetite $\left(\mathrm{Fe}_{3} \mathrm{O}_{4}\right)$ phase to more oxidized magnetite phase, i.e., maghemite $\left(\mathrm{Fe}_{2} \mathrm{O}_{3}\right)$ phase [see Fig. 1(b)]. Figures 2(a) and 2(b)] show the rocking curves at (622) plane for pristine and irradiated thin films. Here again similar results were obtained. At low fluence in the range from $5 \times 10^{10}$ to 5 $\times 10^{11}$ ions $/ \mathrm{cm}^{2}$ the (622) peak is showing slight deformation/shift suggesting that the strain is partially relaxed but the $\mathrm{Fe}_{3} \mathrm{O}_{4}$ phase is retained. In the fluence range of $1 \times 10^{12}-5 \times 10^{12}$ ions $/ \mathrm{cm}^{2}$ there is an appreciable shift in (622) peak indicating the increased relaxation of strain and transformation towards maghemite phase. Finally, with increasing fluence (at $1 \times 10^{13}$ ions $/ \mathrm{cm}^{2}$ ) the (622) peak disappeared which indicates the presence of disorder and complete loss of epitaxial relationship between the substrate and thin film. Using the HRXRD data we have calculated the out-of-
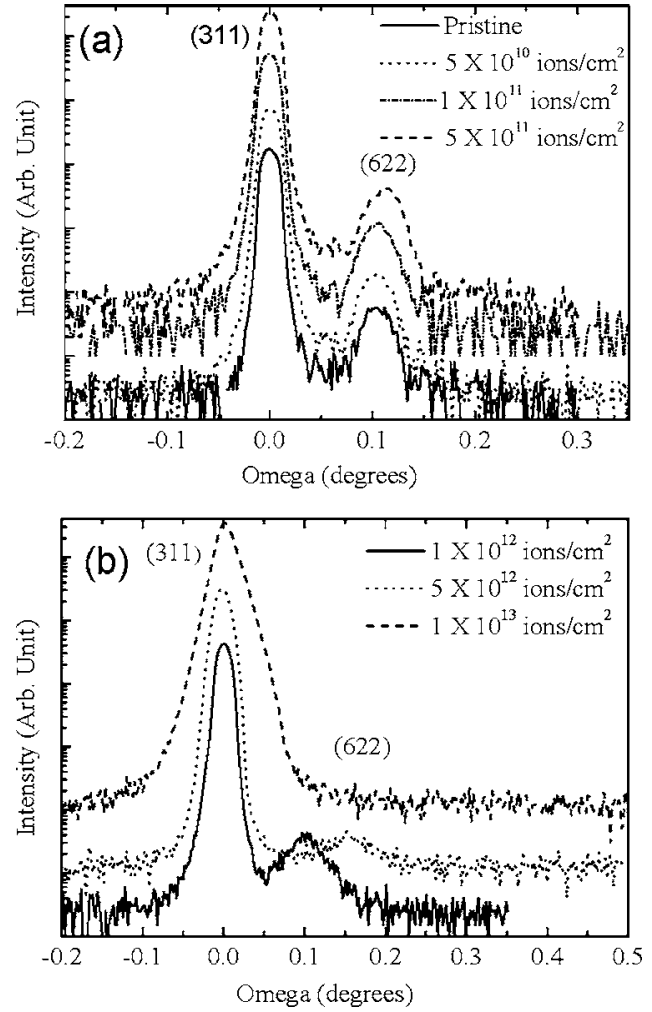

FIG. 2. The $\omega-2 \theta$ rocking curves for pristine and $190 \mathrm{MeV} \mathrm{Ag}^{15+}$ ion irradiated 70-nm-thick $\mathrm{Fe}_{3} \mathrm{O}_{4}$ films (a) with low fluences $\left(5 \times 10^{10}, 1 \times 10^{11}\right.$ and $\left.5 \times 10^{11} \mathrm{ions} / \mathrm{cm}^{2}\right)$ and (b) with higher fluences $\left(1 \times 10^{12}, 5 \times 10^{12}\right.$ and 1 $\times 10^{13}$ ions $/ \mathrm{cm}^{2}$ ) measured for (622) Bragg planes of $\mathrm{Fe}_{3} \mathrm{O}_{4}$. The curves are shifted along the vertical axis for clarity.

plane and in-plane lattice parameters. The detailed analysis of the HRXRD data has been performed. Table I shows the value of lattice parameters for the films irradiated with different fluence values, which indicates that at low fluence the films are partially relaxed, whereas at higher fluence values the mixed phase and finally the disordered maghemite phase appeared. At this point we would like to emphasize that the observed modifications in $\mathrm{Fe}_{3} \mathrm{O}_{4}$ films grown on $\mathrm{MgO}$ substrate are due to the two effects such as (i) SHI induced modifications in $\mathrm{Fe}_{3} \mathrm{O}_{4}$ films and (ii) the modification at the

TABLE I. Summary of HRXRD results; $\Delta \omega$ is the separation between (200) substrate and (400) thin film Bragg planes, out-of-plane lattice parameter $a_{\perp}$, in-plane lattice parameter $a_{\|}$and remarks.

\begin{tabular}{lcccc}
\hline \hline \multicolumn{1}{c}{ Samples } & $\Delta \omega$ & $a_{\perp}(\AA)$ & $a_{\|}(\AA)$ & Remarks \\
\hline Pristine & 0.1652 & 8.3646 & 8.426 & Fully strained \\
$5 \times 10^{10} \mathrm{ions} / \mathrm{cm}^{2}$ & 0.1673 & 8.3638 & 8.426 & Fully strained \\
$1 \times 10^{11} \mathrm{ions} / \mathrm{cm}^{2}$ & 0.1645 & 8.3648 & & Partially relaxed \\
$5 \times 10^{11} \mathrm{ions} / \mathrm{cm}^{2}$ & 0.1680 & 8.3636 & & Partially relaxed \\
$1 \times 10^{12} \mathrm{ions} / \mathrm{cm}^{2}$ & 0.1542 & 8.3670 & & Relaxed + mixed \\
& 0.10 & 8.3925 & & phase \\
& 0.2388 & 8.3376 & & Relaxed + mixed \\
$5 \times 10^{12} \mathrm{ions} / \mathrm{cm}^{2}$ & 0.1105 & 8.3847 & & phase \\
& 0.1816 & 8.3586 & & \\
& 0.2421 & 8.3364 & & Disordered \\
& 0.3300 & 8.3042 & & meghamite phase \\
\hline \multirow{2}{*}{$1 \times 10^{13}$ ions $/ \mathrm{cm}^{2}$} & 0.3475 & 8.2981 & & \\
& & & & \\
\hline \hline
\end{tabular}



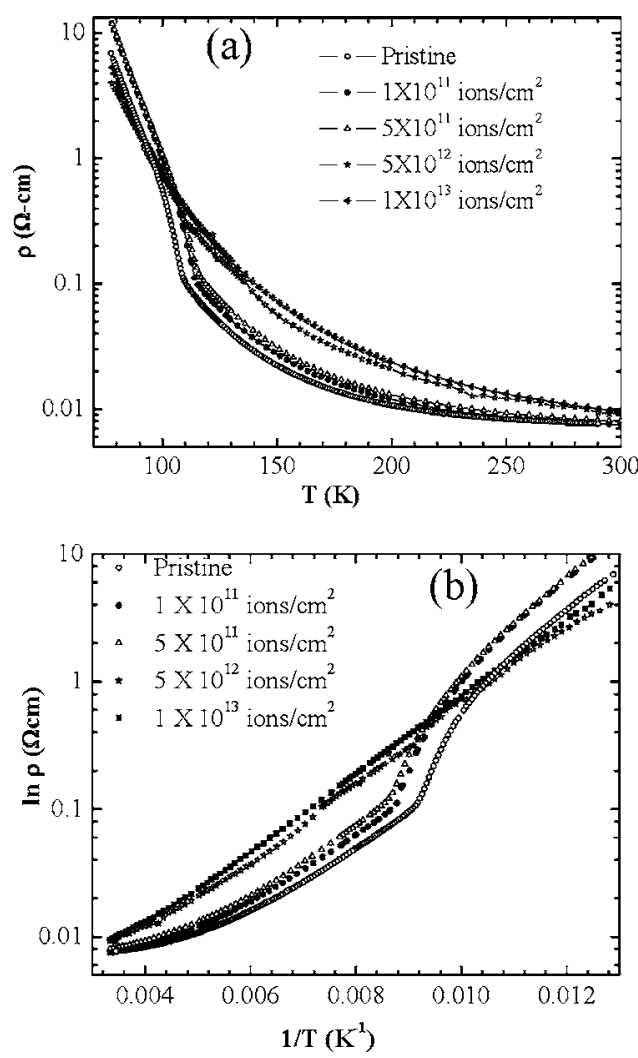

FIG. 3. Resistivity as a function of (a) temperature and (b) inverse of temperature for pristine and $190 \mathrm{MeV} \mathrm{Ag}{ }^{15+}$ ion irradiated 70-nm-thick $\mathrm{Fe}_{3} \mathrm{O}_{4}$ films with fluences $1 \times 10^{11}, 5 \times 10^{11}, 5 \times 10^{12}$ and $1 \times 10^{13} \mathrm{ions} / \mathrm{cm}^{2}$.

films and substrate interface. However, it is hard to separate the contribution of these two effects. We have presented here the overall effect of SHI irradiation on structural magnetic and electrical transport properties of $\mathrm{Fe}_{3} \mathrm{O}_{4}$ thin films.

Figures 3(a) and 3(b) show the resistivity as a function of temperature and inverse of temperature for pristine and $190 \mathrm{MeV} \mathrm{Ag}{ }^{15+}$ ion irradiated $\mathrm{Fe}_{3} \mathrm{O}_{4}$ thin films, respectively. It is clearly evident from Fig. 3 that the Verwey transition temperature $T_{v}$ of these films increases with the ion fluence values from $109 \mathrm{~K}$ (for pristine) to $117 \mathrm{~K}$ for the film irradiated with $5 \times 10^{11}$ ions $/ \mathrm{cm}^{2}$. Our explanation for this result is that the SHI irradiation changes the structure of the anti phase boundaries, possibly even anneals out some of the boundaries. However, at higher fluences the films do not show Verwey transition down to $77 \mathrm{~K}$ and the resistivity value is higher than that of the pristine film again representing that the SHI irradiation induced structural disorder in the film (in agreement to XRD results). It is also evident from the resistivity results that at higher fluences, where the structural transformation takes place, the resistivity is increased. The structural transformation may be the change in the structure at APBs or transformation to meghemite phase. Although the meghemite phase is insulating in nature, these films show semiconducting behavior with finite carrier concentration, which may be due to the disordered maghemite phase or incomplete phase transformation.

The Verwey transition is an important feature of $\mathrm{Fe}_{3} \mathrm{O}_{4}$ system and it represents the setting of long range ordering of $\mathrm{Fe}^{2+}$ and $\mathrm{Fe}^{3+}$ ions at octahedral sites. This ordering is de-

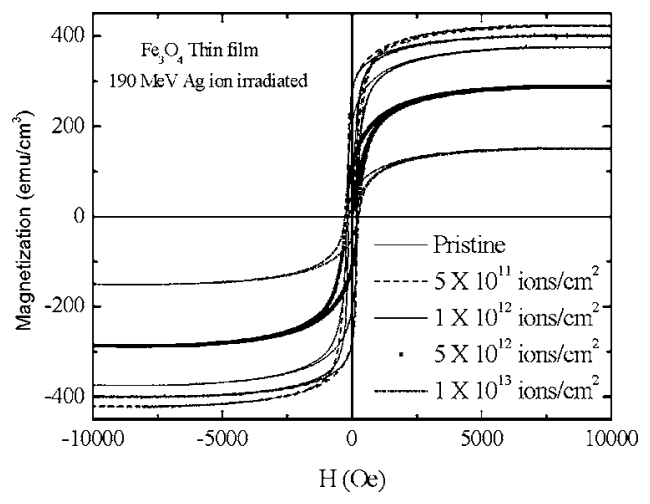

FIG. 4. Isothermal dc magnetization hysteresis curve for pristine and $190 \mathrm{MeV} \mathrm{Ag}{ }^{15+}$ ion irradiated 70-nm-thick $\mathrm{Fe}_{3} \mathrm{O}_{4}$ films with fluences (5 $\times 10^{11}, 1 \times 10^{12}, 5 \times 10^{12}$ and $1 \times 10^{13}$ ions $\left./ \mathrm{cm}^{2}\right)$ at $300 \mathrm{~K}$.

pendent upon various parameters, such as onsite and interionic Coulomb potential and the bandwidth. Both of these parameters are very sensitive to the strain, APB density, and the cation distribution or substitution in $\mathrm{Fe}_{3} \mathrm{O}_{4}$ thin films. As discussed in the light of mean-field approach presented by Brabers, Walz, and Kronmuller ${ }^{6,7}$ and Coey et al., ${ }^{8}$ if the bandwidth is increased and the Coulomb potential is kept constant, the $T_{v}$ will decrease. At a critical value of the ratio $R_{c}=U / W \approx 2.2$ (where $U$ is interionic Coulomb potential and $W$ is bandwidth), the Verwey transition will disappear. This model has been proved and fully in agreement with various experimental situations such as impurity substitution ${ }^{27}$ pressure effect ${ }^{28}$ on Verwey transition, the deviation from the ideal stochiometery ${ }^{29}$ and Verwey transition in lowdimensional systems. ${ }^{5}$ All these experiments proposed the variation of bandwidth and interionic Coulomb interaction and its effect on Verwey transition temperature. It is clearly evident from the results of Figs. 3(a) and 3(b) that SHI irradiation is a good tool to tune the $T_{v}$ to an optimum value. On the other hand, one can say that the SHI irradiation is a tool to tune the bandwidth (in relation to strain, APB density,and structural phase transition) in the $\mathrm{Fe}_{3} \mathrm{O}_{4}$ system depending upon ion energy and fluence values.

Further, to validate our conclusions, the magnetization measurements on pristine and irradiated films were performed. Figure 4 shows the isothermal dc magnetization hysteresis at $300 \mathrm{~K}$ for pristine $\mathrm{Fe}_{3} \mathrm{O}_{4}$ and $190 \mathrm{MeV} \mathrm{Ag}{ }^{15+}$ ion irradiated films. Here again the magnetization for the optimized fluence value $\left(5 \times 10^{11}\right.$ ions $\left./ \mathrm{cm}^{2}\right)$ is higher than that of the pristine film. At fluence in excess of 5 $\times 10^{12}$ ions $/ \mathrm{cm}^{2}$ the magnetization decreases rapidly which is consistent with the formation of iron oxide phases other than magnetite. The films exposed to highest fluence (1 $\times 10^{13}$ ions $/ \mathrm{cm}^{2}$ ) show the lowest saturation magnetization $\left(151 \mathrm{emu} / \mathrm{cm}^{3}\right)$, which is even lower than that of $\gamma-\mathrm{Fe}_{2} \mathrm{O}_{3}$ phase $\left(M_{s}=360 \mathrm{emu} / \mathrm{cm}^{3}\right)$. The magnetization results are also in agreement with the HRXRD and resistivity results. The hysteresis loop magnetization measurements are intrinsically bulk measurements and the model proposed in this article addresses the irradiation induced structural transformation and modification of strain state which consequently affects the cationic arrangements at APBs. To confirm this we have performed the magnetic force microscopy measure- 

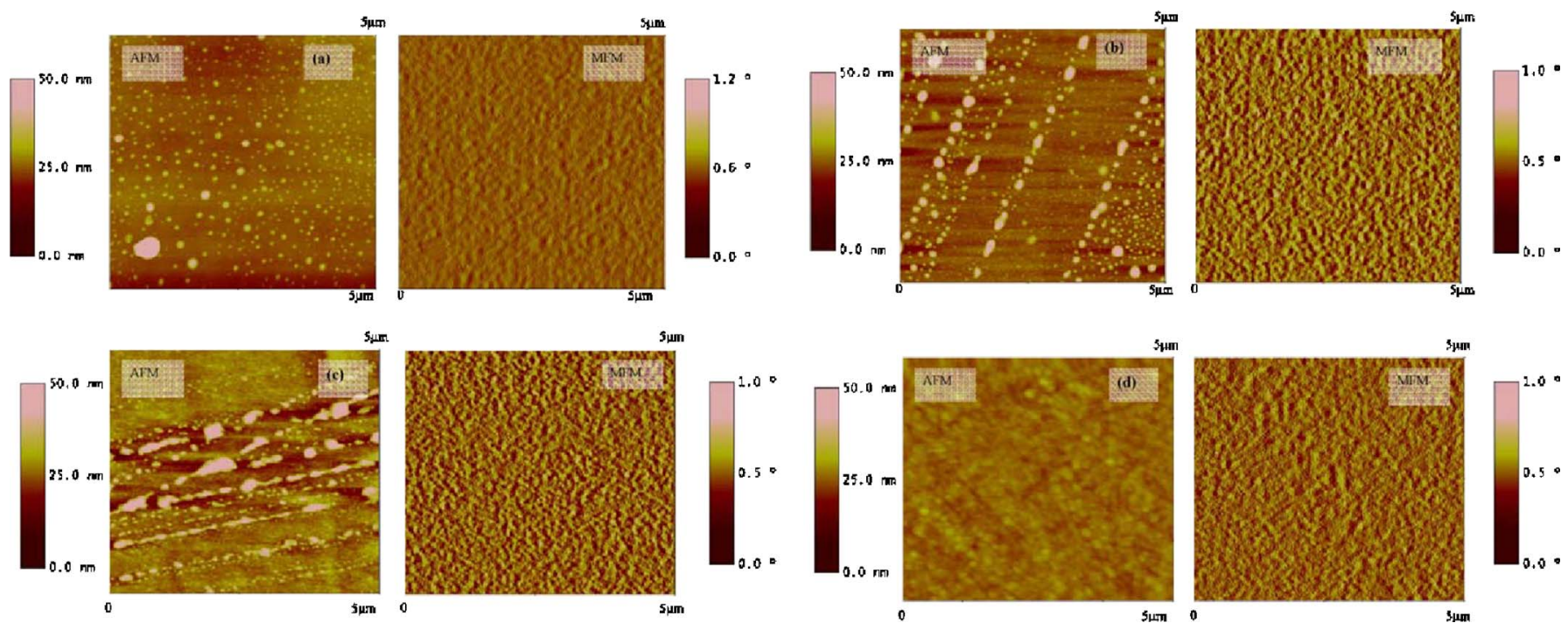

FIG. 5. Atomic force microscopy (AFM) and magnetic force microscopy (MFM) pictures for (a) pristine and $190 \mathrm{MeV}^{\mathrm{Ag}}{ }^{15+}$ ion irradiated 70 -nm-thick $\mathrm{Fe}_{3} \mathrm{O}_{4}$ films with fluences (b) $5 \times 10^{11}$ ions $/ \mathrm{cm}^{2}$, (c) $5 \times 10^{12}$ ions $/ \mathrm{cm}^{2}$ and (d) $1 \times 10^{13}$ ions $/ \mathrm{cm}^{2}$ at room temperature.

ments to study the affect of these microscale transformations on magnetic domains. Figure 5 shows the results of the magnetic force microscopy (MFM) along with the surface morphology measured by atomic force microscopy of pristine and irradiated $\mathrm{Fe}_{3} \mathrm{O}_{4}$ thin films. It is clearly evident that films exposed to $5 \times 10^{11}$ ions $/ \mathrm{cm}^{2}$ fluence show maximum contrast which is consistent with the magnetization data. With further irradiation the contrast is reduced. Recently, Zhou et $a l .^{20,30}$ have attempted to modify the APBs in $\mathrm{Fe}_{3} \mathrm{O}_{4}$ thin films using thermo-chemical treatment, i.e., annealing the films at $250{ }^{\circ} \mathrm{C}$ in air for different time duration, and have observed an increase in saturation magnetization, but at the same time they observed that the Verwey transition was decreased. In the present case, contrary to their results, the Verwey transition temperature and magnetization both increased with SHI irradiation up to an optimum value of ion fluence, which clearly indicates that SHI irradiation has modified the APB structure and possibly annealed out some of the APBs.

From the above presented experimental results, we have conjectured that at low fluence, the swift heavy ion irradiation modifies the strain state of the films and cation distribution in the vicinity of APBs. Whereas, at higher fluence, it introduces structural disorder and transforms the phase from magnetite to maghemite. Here we would like to discuss the variation of Verwey transition with SHI irradiation. As discussed earlier the Verwey transition has a strong dependence on onsite Coulomb potential, interionic Coulomb potential and bandwidth. All these parameters depend upon the valence state of Fe at APB, nature of the strain in the system and local stoichiometry. Our results clearly indicate that by selecting appropriate irradiation parameters, i.e., by keeping the electronic energy loss below the threshold value of columnar defect formation, we are able to tune the material's properties to get an optimum Verwey transition temperature and the maximum magnetization in this system. Finally, we have demonstrated the phase transformation by just varying the ion fluence. To summarize, the SHI irradiation is a tool to modify the various parameters of these materials in a controlled fashion.

${ }^{1}$ M. Ziese, Rep. Prog. Phys. 65, 143 (2002).

${ }^{2}$ F. Walz, J. Phys.: Condens. Matter 14, R285 (2002).

${ }^{3}$ E. W. J. Verwey, Nature (London) 144, 327 (1937).

${ }^{4}$ E. W. J. Verwey and P. W. Haayman, Physica (Amsterdam) 8, 979 (1941).

${ }^{5}$ I. V. Shvets, G. Mariotto, K. Jordan, N. Berdunov, R. Keantor, and S. Murphy, Phys. Rev. B 70, 155406 (2004).

${ }^{6}$ J. H. V. J. Brabers, F. Walz, and H. Kronmuller, J. Phys.: Condens. Matter 12, 5437 (2000).

${ }^{7}$ J. H. V. J Brabers, F. Walz, and H. Kronmuller, J. Phys.: Condens. Matter 11, 3679 (1999).

${ }^{8}$ J. M. D. Coey, I. V. Shvets, R. Wiesendanger, and H. J. Gütherodt, J. Appl. Phys. 73, 6742 (1993)

${ }^{9}$ J. M. Gaines, P. J. H. Bloemen, J. T. Kohlhepp, C. W. T. Bulle-Lieuwma, R. M. Wolf, A. Reinders, R. M. Jungblut, P. A. A. van der Heijden, J. T. W. M. van Eemeren, J. aan de Stegge, and W. J. M. de Jonge, Surf. Sci. 373, 85 (1997).

${ }^{10}$ D. T. Margulies, F. T. Parker, M. L. Rudee, F. E. Spada, J. N. Chapman, P. R. Aitchison, and A. E. Berkowitz, Phys. Rev. Lett. 79, 5162 (1997).

${ }^{11}$ T. Himba, F. C. Voogt, L. Niesen, P. A. A. van der Heijden, W. J. M. de Jonge, J. J. T. M. Donkers, and P. J. van der Zaag, J. Appl. Phys. 85, 5291 (1999).

${ }^{12}$ W. Esrenstein, T. T. M. Palstra, T. Himba, and S. Celotto, Phys. Rev. B 68, 014428 (2003).

${ }^{13}$ D. T. Margulies, F. T. Parker, F. E. Spada, R. S. Goldman, J. Li, R. Sinclair, and A. E. Berkowitz, Phys. Rev. B 53, 9175 (1996).

${ }^{14}$ F. C. Voogt, T. T. M. Palstra, L. Niesen, O. C. Rogojanu, M. A. Janes, and T. Himba, Phys. Rev. B 57, R8107 (1998).

${ }^{15}$ S. Kale, S. M. Bhagat, S. E. Lofland, T. Scabarozi, S. B. Ogale, A. Orozco, S. R. Shinde, T. Venkatesan, B. Mercey, and W. Prellier, Phys. Rev. B 64, 205413 (2001).

${ }^{16}$ S. K. Arora, R. G. S. Sofin, I. V. Shvets, R. Kumar, M. Wasi Khan, and J. P. Srivastava, J. Appl. Phys. 97, 10C310 (2005).

${ }^{17}$ R. Kumar, R. J. Choudhary, S. I. Patil, S. Husain, J. P. Srivastava, S. P. Sanyal, and S. E. Lofland, J. Appl. Phys. 96, 7383 (2004).

${ }^{18}$ S. Khatua, P. K. Mishra, R. Kumar, D. C. Kundaliya, D. Buddhikot, R. Pinto, and S. K. Malik, J. Appl. Phys. 96, 7403 (2004).

${ }^{19}$ R. J. Choudhary, R. Kumar, S. Husain, J. P. Srivastava, S. I. Patil, and S. K. Malik, Appl. Phys. Lett. 82, 222501 (2005).

${ }^{20}$ Y. Zhou, X. Zin, and I. V. Shvets, J. Appl. Phys. 95, 7357 (2004).

${ }^{21}$ J. F. Ziegler, J. P. Biersack, and U. Littmerk, Stopping Power and Ranges of Ions in Matter (Pergamon Press New York, 1985).

${ }^{22}$ J. M. Costantini, F. Studer, and M. Toulemonde, Mater. Sci. Eng., A 253, 121 (1998). 
${ }^{23}$ G. Szenes, Phys. Rev. B 51, 8026 (1995), and references therein.

${ }^{24}$ D. Lesuer and A. Dunlop, Radiat. Eff. Defects Solids 126, 163 (1993).

${ }^{25}$ K. Balakrishnan, S. K. Arora, and I. V. Shvets, J. Phys.: Condens. Matter 16, 5387 (2004).

${ }^{26}$ J. W. Mathew and A. E. Blakeslee, J. Cryst. Growth 27, 118 (1973).

${ }^{27}$ V. A. M. Brabers, F. Walz, and H. Kronmuller, Phys. Rev. B 58, 14163
(1998).

${ }^{28}$ G. K. Rozenberg, G. R. Hearne, and M. P. Pasternak, Phys. Rev. B 53, 6482 (1996)

${ }^{29}$ Z. Kakol and J. M. Honig, Phys. Rev. B 40, 9090 (1989).

${ }^{30}$ Y. Zhou, C. McEvoy, and I. V. Shvets, J. Magn. Magn. Mater. 290-291, 1033 (2005). 